\title{
Efficacy of web-based self-management interventions for depressive symptoms: a meta-analysis of randomized controlled trials
}

Yue Pang®, Xin Zhang, Ruitong Gao, Linqi Xu, Meidi Shen, Hongyu Shi, Yuewei Li and Feng Li* (D)

\begin{abstract}
Background: The incidence of depression is increasing worldwide. Depression can lead to poor physical health and even suicide. However, in high-income countries, only about $50 \%$ of the people with depression receive appropriate therapy, and the detection rate of depression in low- and middle-income countries is relatively lower. Web-based self-management enables remote treatment and solves the problem of insufficient psychological treatment resources. Many past studies have evaluated the effectiveness of web-based self-management of depression, but there has been no synthesis of evidence. Therefore, this study conducted a meta-analysis of the effectiveness of web-based self-management for depressive symptoms.
\end{abstract}

Method: Six electronic databases (Cochrane Central Register of Controlled Trials, PubMed, Web of Science, Embase, CINAHL, and PsycINFO) were searched in September 2020. All literature referring to the effects of web-based selfmanagement on depression were shortlisted by performing the medical subject headings (MeSH) search combined with a text word search.

Results: A total of 18 eligible randomized controlled trials were identified, and the results from 3055 participants were consolidated. The web-based self-management group exhibited a greater reduction in depressive symptoms than the control group ( $\mathrm{g}=-0.46 ; 95 \% \mathrm{Cl}: 0.62,0.30$ ), and there was no evidence of publication bias. Subgroup analysis revealed that patients with moderate-to-severe depression benefited from web-based self-management interventions. In terms of interventions, those based on cognitive behavioral therapy (CBT) were highly effective. We noted that the longer the intervention time, the better was the improvement in the status of depression. Furthermore, it was established that participants who communicated with therapists and showed greater adherence to the intervention experienced significant improvement in their symptoms. The results of the intervention group were better than those of the waiting-list, treatment-as-usual, and online psychoeducation groups.

Conclusions: Web-based self-management is a promising therapy for depression. Future research should aim to refine these aspects of the intervention to achieve a beneficial impact.

Keywords: Depression, Web-based, Self-management, Meta-analysis

\footnotetext{
*Correspondence: fli@jlu.edu.cn

School of Nursing, Jilin University, Changchun, China
}

(c) The Author(s). 2021 Open Access This article is licensed under a Creative Commons Attribution 4.0 International License, which permits use, sharing, adaptation, distribution and reproduction in any medium or format, as long as you give appropriate credit to the original author(s) and the source, provide a link to the Creative Commons licence, and indicate if changes were made. The images or other third party material in this article are included in the article's Creative Commons. licence, unless indicated otherwise in a credit line to the material. If material is not included in the article's Creative Commons licence and your intended use is not permitted by statutory regulation or exceeds the permitted use, you will need to obtain permission directly from the copyright holder. To view a copy of this licence, visit http://creativecommons.org/licenses/by/4.0/ The Creative Commons Public Domain Dedication waiver (http://creativecommons.org/publicdomain/zero/1.0/) applies to the data made available in this article, unless otherwise stated in a credit line to the data. 


\section{Introduction}

According to the World Health Organization's World Mental Health Survey (WMH), the lifetime prevalence of severe depression varies widely across country, with 19.2\% people in the United States, 21\% in France, 17.9\% in the Netherlands, $6.6 \%$ in Japan, and $18.4 \%$ in Brazil being afflicted by this condition [1]. On an average, the lifetime prevalence of depression was noted to be higher in high-income countries (14.6\%) than in low- and middle-income countries (11.1\%) [2]. The high incidence of depression means that it is common, with the frequency of almost one in five people experiencing a depressive episode at some point in their lives. Depressive symptoms occur in 1 in 10 patients on average in primary care settings [3].

In addition to aggravating personal suffering, depression has been linked to poor physical health, impaired social functioning, and even suicide. Hence, the illness imposes a great burden on both individuals and society, especially considering the stress of clinical psychotherapy [4]. The presence of depression significantly influences the prevalence, costs, and outcomes of several common comorbidities [5]. The results of a Hong Kong-based cohort study suggest that depressive symptoms are likely to be independent causes of suicide [6,7].

Depression is treatable, and the current treatment consists mainly of antidepressant medications and psychotherapy. Despite a large number of high-quality research evidence implying that both the methods are effective, only about half of the people with depression receive proper treatment $[8,9]$. Nearly $50 \%$ of the world's population lives in countries with less than one psychiatrist per 100,000 individuals [10]. Obstacles are rampant in both treatments. First of all, drug treatment can lead to low compliance owing to the stigma attached to it, especially among young patients. Second, concerning psychotherapy, there is a dearth of trained mental health personnel and psychological treatment institutions, which are far from meeting the needs of the patients $[11,12]$. To sum up, although both interventions are effective, their implementation rate is low. Therefore, interventions that are both effective and easy to implement are urgently needed.

In this scenario, web-based self-management of depression may be a promising solution. To simplify, selfmanagement of depression indicates that an individual is an active self-manager. Moreover, with or without the support of healthcare professionals, this individual can actively treat his/her depression gradually on an everyday basis, by performing activities such as obtaining disease information, drug management, symptom management, lifestyle changes, and actively seeking social support and communication, thereby reducing the possibility of relapse and tending toward a healthier life [13]. A concept similar to self-management is self-help, and most of the currently known self-help approaches for treating depression involve repackaged CBT-based technologies that aim to treat the acute symptoms of depression. Self-management of depression involves proactively learning ways to manage the depression over a longer period, which can teach the patients more useful skills that can continue to work above and beyond the short-term relief that may be otherwise gained from the conventional self-help strategies. Self-help for depression can be seen as self-management with short-term effects; hence, this manuscript also included articles on self-help for depression in the metaanalysis.

To understand the advantages of self-management of depression by using the Internet, when compared with face-to-face or telephonic intervention, web-based selfmanagement involves no restrictions on cost, personnel, or resources and can therefore improve the efficiency of intervention, necessitating less therapist time [14].

The existing randomized controlled trials have asserted that self-management provided through the internet can help improve the treatment for patients with moderate and severe depression [15-18]. This intervention has long-term effects and is sustainable for 3 years [19]. Research has indicated that web-based self-management interventions can also prevent the recurrence of depression [20]. However, certain studies have alluded that webbased, guided self-help interventions are less effective than the standard nursing control group [21, 22]. Another study has demonstrated that Internet-based problemsolving therapy is not more effective in reducing symptoms of depression than that by receiving an unguided self-help book during the waiting list period at outpatient mental health clinics [23]. The current consensus on whether web-based self-management is effective for alleviating depressive symptoms remains debatable and hence unclear. Previous meta-analyses have shown that selfguided internet-based cognitive behavioral therapy (iCBT) is effective in treating depressive symptoms [24]. This meta-analysis aimed to expand the theoretical scope of intervention by including studies based on CBT, as well as on other theories, such as problem-solving treatment (PST), positive psychotherapy (PPT), emotion-focused therapy, and interpersonal therapy (IPT). Moreover, this study not only analyzed the effectiveness of intervention from the theoretical perspective but also considered the influence of intervention time and guidance.

In summary, the effectiveness of web-based selfmanagement interventions for depression is controversial, hence it makes sense to conduct this meta-analysis. This study aimed to assess the effectiveness of selfmanagement through the internet in reducing depressive symptoms. The outcome variables include the changes in the depression scores from baseline to postintervention, measured using any of the well-validated 
depression scales. We also attempted to use subgroup analysis to explore which aspects of web-based selfmanagement intervention have a high or low curative effect on depressive symptoms. The results of this study are expected to provide a preliminary direction for the formulation of such interventions in the future. The findings are also likely to guide treatment selection and further research in this field.

\section{Methods}

\section{Registration}

This meta-analysis abided by the PRISMA statement $[25,26]$. To eliminate the bias of the researchers, the search strategy, inclusion criteria, data extraction, and pre-planned subgroup analysis were strictly monitored by the PROSPERO system review through a registration agreement (CRD42020223172).

\section{Literature search strategies}

We searched the following databases from the establishment of the database to September 21, 2020: Cochrane Central Register of Controlled Trials, PubMed, Web of Science, Embase, CINAHL, and PsycINFO. All literature related to the effects of web-based self-management interventions on patients with depression was obtained by using the medical subject headings $(\mathrm{MeSH})$ search combined with a text word search. The specific search strategy is depicted in Additional file 1. The reviews on related topics were searched and analyzed, all the references in the relevant articles were manually searched, and the studies that met the inclusion criteria of this research were included. The literature search process was simultaneously carried out by two authors, and if any differences emerged, the third author resolved them. The search results were imported into Endnote, and some preliminary filtering was done by browsing the titles and abstracts.

\section{Eligibility criteria \\ Inclusion criteria}

We only included randomized controlled trials published in the English language from the establishment of the database until September 21, 2020. The minimum intervention period for inclusion in this study was 4 weeks. The literature selection process involved three independent researchers who evaluated the quality of the articles and resolved any differences through discussion.

The participants included in this study were required to meet the following criteria: depression was diagnosed by a physician based on DSM-IV, ICD-10, or assessed by any well-validated depression scales (BDI-II, CES-D, MADRS$\mathrm{S}$, and PHQ-9); no cognitive impairment or any other mental illness. The purpose of this study was to examine the impact of web-based self-management interventions on the participants' depressive symptoms. Hence, there were no restrictions on any other clinical or demographic characteristics of the eligible participants.

Only web-based self-management interventions were included in this study, such as the imparting of selfmanagement skills through websites, programs, and interactive games. Self-management intervention should include the introduction of depression knowledge; treatment to improve behavior, cognition, and emotion; methods to prevent recurrence; methods to improve sleep and physical health, and the necessary knowledge of medication. In this study, both therapist-contacting and non-contacting studies during the intervention were included.

The control groups in this study included the waitinglist group, treatment-as-usual group, and an online psychoeducation group. The waiting list group did not receive care until the post-test data were collected from both the groups. The treatment-as-usual group was provided the usual mental health care, while the online psychoeducation group was provided an online psychological education about depression.

At least one primary or secondary outcome measure illustrating the depressive symptoms was used.

\section{Exclusion criteria}

(1) Duplicate articles were excluded.

(2) Meeting reports, guidelines, newspapers, and summaries were excluded.

(3) Randomized controlled trials with no control group were excluded.

(4) If the implementation of self-management was not clearly stated in the intervention, the research was excluded.

\section{Study selection and data extraction}

After literature screening, two independent researchers extracted data from published reports, and they filled identical data extraction forms. In case of disagreement, the problem was solved through discussion or contact with the author.

Each article made use of the data extraction table to retrieve the following data:

(1) Study information (investigator, year, country, sample size, average age of the participants, diagnostic information or relevant inclusion criteria, study duration, adherence to the interventions, dropout rate, and trial quality)

(2) Intervention functions (website/application name, self-managed program content, and any other intervention components)

(3) Detailed information on the control group. 
(4) Impact on depressive symptoms (the total depressive symptoms were scored before and after by using any clinically validated assessment scale)

\section{Statistical analyses}

All the included studies were assessed for quality according to the bias risk tool mentioned in the Cochrane Intervention System Assessment Manual. The research quality was assessed in terms of seven aspects (random sequence generation, allocation concealment, blinding of participants and personnel, blinding of outcome data, incomplete outcome data, selective reporting, and other bias), and the risk of each type of prejudice was classified as "unclear," "low," or "high." [27] The results were entered in Review Manager 5.3 software (Nordic Cochrane Center, Copenhagen, Denmark) for generating bias risk graphs and bias risk summaries.

In this study, the statistical software Stata version 16.0 (Stata Corporation, College Station, TX, USA) was used to analyze the impact of web-based selfmanagement intervention on the participants' depression status. The data were in the form of continuous variables, which were expressed as mean \pm standard deviation. $\mathrm{Q}$ test and $\mathrm{I}^{2}$ statistics were used to test the heterogeneity. Significant differences in Q values indicated that the selected research papers were heterogeneous, and $\mathrm{I}^{2}$ values of $25.0,50.0$, and $75.0 \%$ indicated low medium, and high heterogeneity, respectively. Considering the sampling errors between and within groups, the fixed effect model was employed for meta-analysis of homogeneous research, while the random effect model was utilized for metaanalysis of heterogeneous research. Standardized mean difference (as Hedges' g) and 95\% confidence intervals (CI) were exploited to express the effectiveness of the self-management intervention in treating depression when compared with the control group. Furthermore, Egger's test and trim-and-fill analysis were used to evaluate any publication bias. $p<0.05$ (two-sided) was considered statistically significant.

A pre-planned subgroup analysis was conducted to examine whether the effects of web-based selfmanagement interventions on depressive states were different from those of the waiting list, treatment-as-usual and online psychoeducation groups. In addition, a series of exploratory investigations of subgroups and metaregression analysis were done to ascertain the factors that may influence the effectiveness of web-based selfmanagement interventions, including the detailed information on the sample (e.g., participants' depression severity) and intervention characteristics (e.g., the theory of intervention support, the duration of intervention, and presence of therapist guidance).

\section{Results}

Study selection

After careful screening of six databases, we obtained a total of 789 records. We eliminated 150 duplicate records and identified 639 relevant articles. In this process, by browsing the titles and abstracts, we excluded 392 articles that did not meet the inclusion criteria of this study and identified 247 articles for possible inclusion. Subsequently, by reading the full text, we excluded 48 articles that did not conform to depression, 87 articles that did not conform to intervention measures, seven articles that did not have a control group, 11 articles that did not conform to outcomes, 71 articles that were not randomized controlled trials, three articles for which full text could not be retrieved, and two articles that were not in English. Finally, we included 18 randomized controlled trials for analysis [15-18, 21-23, 28-38]. The article screening process is illustrated in Fig. 1.

\section{Study characteristics}

Table 1 shows the details of each study. A total of 3055 participants were included from 18 studies published between 2005 and 2020. The adherence to the interventions and drop-out in each study are indicated in Table 1. Both the intervention and control groups comprised individuals with depression. Web-based self-management intervention was compared with the waiting list, treatment-as-usual, and online psychoeducation groups. The duration of the intervention ranged from 4 weeks to 12 weeks. The outcome measure was changed in depressive symptoms, which happened to be the primary outcome in 18 articles and the secondary outcome in one article. The Beck Depression Scale-II (BDI-II) was used in seven studies [15, 17, 28, 29, 31, 36, 38]. The Epidemiology Research Center Depression Scale (CESD) was used in six studies [16, 21-23, 30, 33]. The Patient Health Questionnaire depression module (PHQ-9) was used in one study [35]. The Patient Health Questionnaire (PHQ8) was used in one study [37]. The Hamilton Depression Scale (HRSD) was used in one study [18]. The Hospital Anxiety and Depression Scale (HADS) was used in one study [34]. The Montgomery-Eisenberg Depression Scale (MADRS-S) was used in one study [19]. The Modified Childhood Depression Rating Scale (CDRS-R) was used in one study [32].

\section{Risk of bias within studies}

As portrayed in Fig. 2, we used the Cochrane risk bias assessment tool to ascertain the quality of the 18 randomized controlled trials. The random sequence generation risk of the articles was found below. Eleven studies $[15,18,21-23,28,32,34-36,38]$ explicitly mentioned that allocation was hidden. The most common risk factor for bias in this meta-analysis was insufficient blindness on the part of the researchers or participants, with 


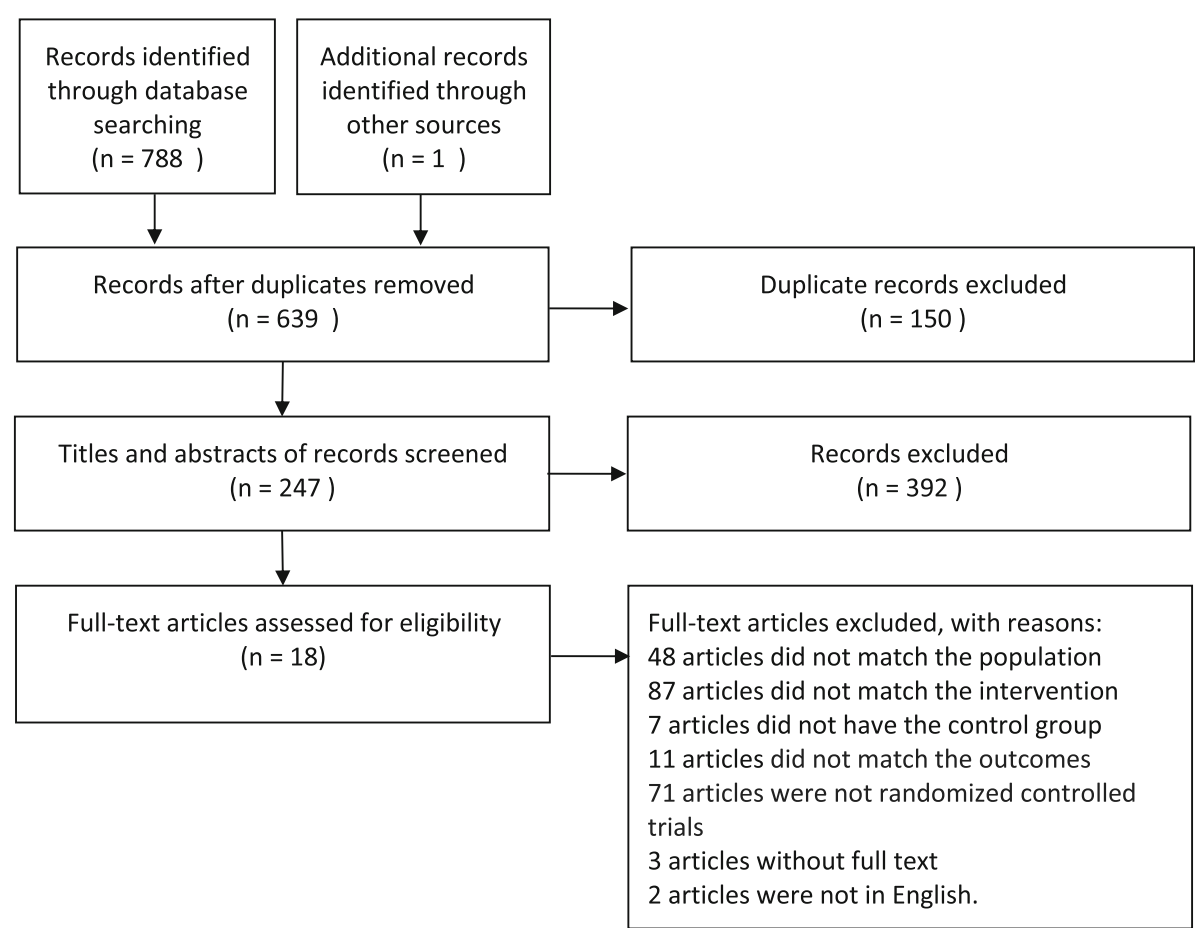

Fig. 1 PRISMA Flow Chart of Study Selection

the researchers and participants not knowing the allocation of interventions in only four $[15,16,21,30]$ of the 18 studies. The measurement bias was not clear in four articles [16, 22, 31, 37], and one [15] was defined as high risk. Three of the articles [15, 33, 37] did not provide complete data. All the studies were low-risk in terms of selective reporting and other biases.

\section{The overall impact of web-based self-management interventions on depressive symptoms}

Figure 3 illustrates the combined effect of web-based self-management interventions on depressive symptoms as well as the individual effects of each study. The results from the random-effects model indicated that the webbased self-management intervention had a positive effect on the reduction of depressive symptoms when compared with the control group $(N=3055, \mathrm{~g}=-0.46$; $95 \%$ CI: $-0.62,-0.30 ; p<0.05)$. Despite the presence of some heterogeneity between the studies $\left(\mathrm{I}^{2}=75.59 \% ; p<0.01\right)$, there was no evidence of publication bias (Egger test: $P>|t|=0.333>0.05)$. The funnel plots are presented in Additional file 3.

\section{Additional analysis}

\section{Population characteristics and effects on depressive} symptoms

To determine the population in which web-based selfmanagement interventions were most effective and to ascertain the sources of heterogeneity in this metaanalysis, we conducted a subgroup analysis based on the participants' baseline depression severity (Fig. 3B). They were divided into mild depression groups and moderateto-severe depression groups. The results implied that the combined effects on the moderate-to-severe depression group were statistically significant $(\mathrm{g}=-0.54$; $95 \% \mathrm{CI}$ : $0.83,-0.24 ; p=0.00$ ); however, the combined effect on the mild depression group was not statistically significant ( $\mathrm{g}=-0.57 ; 95 \% \mathrm{CI}:-1.27,0.13 ; p=0.01$ ). However, as the difference between the groups was not statistically significant, inter-group comparisons were not appropriate. Subgroup analysis indicated that the heterogeneity of this study may be due to the differences in the severity of depression at baseline $\left(\mathrm{I}^{2}=82.96 \% ; p=0.00\right)$.

\section{Intervention characteristics and effects on depressive symptoms}

To further understand which aspects of the web-based self-management intervention were effective in tackling depression, we conducted subgroup analysis according to the different characteristics of intervention (such as the theory on which the intervention was based, the duration of intervention, and the contact of the therapist). The different characteristics of the interventions and the results of all subgroup comparisons are presented in Table 2 in a detailed manner. (The forest plot for 
Table 1 Details of Included Studies

\begin{tabular}{|c|c|c|c|c|c|c|c|c|}
\hline $\begin{array}{l}\text { Author/ } \\
\text { country/ } \\
\text { year }\end{array}$ & Sample type & $\begin{array}{l}\text { Adherence } \\
\text { to the } \\
\text { interventions }\end{array}$ & $\begin{array}{l}\mathrm{N} \\
\text { dropout(\%) }\end{array}$ & $\begin{array}{l}\text { Age } \\
\text { (years,mean) }\end{array}$ & Intervention design & Control group & $\begin{array}{l}\text { Treatment(T) } \\
\& \text { follow } \\
\text { up(F) }\end{array}$ & $\begin{array}{l}\text { Outcome } \\
\text { (mean,SD) }\end{array}$ \\
\hline $\begin{array}{l}\text { Andersson, } \\
\text { G.et al./ } \\
\text { Sweden/ } \\
2005[28]\end{array}$ & $\begin{array}{l}\text { Major } \\
\text { depression } \\
\text { (for the full } \\
\text { CIDI) + mild- } \\
\text { to-moderate } \\
\text { depression } \\
\text { (MADRS-S) }\end{array}$ & $\begin{array}{l}36 / 57 \\
(63.16 \%)\end{array}$ & $\begin{array}{l}117 / 32 \\
(27.35 \%)\end{array}$ & $\begin{array}{l}\text { l:36.4 (11.5) C: } \\
36.3(9.9)\end{array}$ & $\begin{array}{l}\text { The cognitive- } \\
\text { behavioural self-help } \\
\text { treatment: study on the } \\
\text { website + the therapist } \\
\text { gave an e-mail feed- } \\
\text { back + the discussion } \\
\text { groups }\end{array}$ & $\begin{array}{l}\text { A web-based dis- } \\
\text { cussion group }\end{array}$ & T:10W F:6 m & $\begin{array}{l}\text { BDI: I: } 12.2 \\
(6.8) C: \\
19.5(8.1)\end{array}$ \\
\hline $\begin{array}{l}\text { Berger, } \\
\text { T.et al./ } \\
\text { Switzerland } \\
\text { and } \\
\text { Germany/ } \\
2011 \text { [29] }\end{array}$ & $\begin{array}{l}\text { Moderate to } \\
\text { severe } \\
\text { depression } \\
\text { (BDI-II) }\end{array}$ & $25 / 25(100 \%)$ & $51 / 4(7.84 \%)$ & $38.8(14.0)$ & $\begin{array}{l}\text { The web-based self- } \\
\text { help program } \\
\text { (Deprexis) + scheduled } \\
\text { e-mail contact with a } \\
\text { therapist }\end{array}$ & Waiting list & $T: 10 w$ F:6 m & $\begin{array}{l}\text { BDI-II: I: } \\
17.3(10.2) \\
\text { C: } 28.5 \\
(9.4)\end{array}$ \\
\hline $\begin{array}{l}\text { Boele, F. } \\
\text { W.et al./ } \\
\text { Netherlands/ } \\
2018 \text { [21] }\end{array}$ & $\begin{array}{l}\text { At least mild } \\
\text { depressive } \\
\text { symptoms } \\
\text { (CES-D) }\end{array}$ & $\begin{array}{l}19 / 45 \\
(42.22 \%)\end{array}$ & $\begin{array}{l}89 / 36 \\
(40.45 \%)\end{array}$ & $\begin{array}{l}\text { l:43.58 (11.69) } \\
\text { C:46.43 } \\
(12.28)\end{array}$ & $\begin{array}{l}\text { Online guided self-help } \\
\text { course }\end{array}$ & Waiting list & $T: 6 w$ F:12 m & $\begin{array}{l}\text { CES-D: I: } \\
18.84(6.4) \\
\text { C:23.09 } \\
(7.1)\end{array}$ \\
\hline $\begin{array}{l}\text { Bolier, } \\
\text { L.et al./ } \\
\text { Netherlands/ } \\
2013 \text { [30] }\end{array}$ & $\begin{array}{l}\text { mild to } \\
\text { moderate } \\
\text { depressive } \\
\text { symptoms } \\
\text { (CES-D) }\end{array}$ & $\begin{array}{l}95 / 143 \\
(66.43 \%)\end{array}$ & $\begin{array}{l}284 / 70 \\
(24.65 \%)\end{array}$ & $43.2(11.8)$ & $\begin{array}{l}\text { An online self-help } \\
\text { intervention (Psyfit): } \\
\text { psycho-education + } \\
\text { practical exercise }\end{array}$ & Waiting list & $\mathrm{T}: 2 \mathrm{~m} F: 6 \mathrm{~m}$ & $\begin{array}{l}\text { CES-D: I: } \\
13.67 \\
(6.69) \mathrm{C:} \\
15.39 \\
(7.64)\end{array}$ \\
\hline $\begin{array}{l}\text { Bücker, } \\
\text { L.et al./ } \\
\text { Germany/ } \\
2019 \text { [31] }\end{array}$ & $\begin{array}{l}\text { Self-reported } \\
\text { depressive } \\
\text { symptoms }\end{array}$ & $\begin{array}{l}47 / 64 \\
(73.44 \%)\end{array}$ & $\begin{array}{l}127 / 24 \\
(18.90 \%)\end{array}$ & $\begin{array}{l}\text { l:44.02 (10.90) } \\
C: 48.02 \\
(10.95)\end{array}$ & $\begin{array}{l}\text { An internet-based self- } \\
\text { help program (MOOD) }\end{array}$ & $\begin{array}{l}\text { Care as usual } \\
\text { (CAU) }\end{array}$ & $\mathrm{T}: 6 \mathrm{~W}$ & $\begin{array}{l}\text { BDI-II: I: } \\
20.36 \\
(14.70) \mathrm{C}: \\
18.68 \\
(12.79)\end{array}$ \\
\hline $\begin{array}{l}\text { Ebert, D. } \\
\text { D.et al./ } \\
\text { Germany/ } \\
2017 \text { [16] }\end{array}$ & $\begin{array}{l}\text { at least } \\
\text { moderate } \\
\text { symptoms of } \\
\text { depression } \\
\text { (CES-D) }\end{array}$ & $\begin{array}{l}129 / 130 \\
(99.23 \%)\end{array}$ & $\begin{array}{l}260 / 4 \\
(0.02 \%)\end{array}$ & $50.8(11.8)$ & $\begin{array}{l}\text { A guided Internet- } \\
\text { based self-help inter- } \\
\text { vention (GET.ON Mood } \\
\text { Enhancer Diabetes) }\end{array}$ & $\begin{array}{l}\text { Treatment as } \\
\text { usual + online } \\
\text { psychoeducation } \\
\text { about depression }\end{array}$ & $\mathrm{T}: 2 \mathrm{~m} F: 6 \mathrm{~m}$ & $\begin{array}{l}\text { CES-D: I: } \\
21.1(8.8) \\
\text { C:28.9 (8.7) }\end{array}$ \\
\hline $\begin{array}{l}\text { Eysenbach, } \\
\text { Gunther } \\
\text { et al./ } \\
\text { Netherlands/ } \\
2014\end{array}$ & $\begin{array}{l}\text { depressive } \\
\text { symptoms } \\
\text { (CES-D) }\end{array}$ & $\begin{array}{l}75 / 116 \\
(64.66 \%)\end{array}$ & $\begin{array}{l}231 / 60 \\
(25.97 \%)\end{array}$ & $43.4(9.2)$ & $\begin{array}{l}\text { A worker-directed, } \\
\text { Web-based, guided } \\
\text { self-help intervention } \\
\text { (Happy@Work) }\end{array}$ & $\begin{array}{l}\text { Care as usual } \\
\text { (CAU) }\end{array}$ & $\mathrm{T}: 2 \mathrm{~m}$ F:12 m & $\begin{array}{l}\text { CES-D: I: } \\
15.8(10.6) \\
\text { C:18.3 (9.1) }\end{array}$ \\
\hline $\begin{array}{l}\text { Johansson, } \\
\text { R.et al./ } \\
\text { Sweden/ } \\
2012 \text { [15] }\end{array}$ & $\begin{array}{l}\text { Mild, } \\
\text { moderate, and } \\
\text { major } \\
\text { depression } \\
\text { (MADRS-S) + } \\
\text { major } \\
\text { depressive } \\
\text { disorder } \\
\text { (DSM-IV) }\end{array}$ & $\begin{array}{l}42 / 46 \\
(91.30 \%)\end{array}$ & $92 / 4(4.35 \%)$ & $45.6(14.0)$ & $\begin{array}{l}\text { A guided self-help psy- } \\
\text { chodynamic treatment } \\
\text { (SUBGAP) + online sup- } \\
\text { port from a therapist }\end{array}$ & $\begin{array}{l}\text { Non-directive } \\
\text { online supportive } \\
\text { treatment }\end{array}$ & T:10w F:10 m & $\begin{array}{l}\text { BDI-II: I: } \\
11.48 \text { (7.8) } \\
\text { C:20.22 } \\
(7.8)\end{array}$ \\
\hline $\begin{array}{l}\text { Kenter, } \\
\text { Robin Maria } \\
\text { Francisca } \\
\text { et al./ } \\
\text { Netherlands/ } \\
2016 \text { [23] }\end{array}$ & $\begin{array}{l}\text { major } \\
\text { depressive } \\
\text { disorder } \\
\text { (DSM-IV) }\end{array}$ & $\begin{array}{l}94 / 136 \\
(69.12 \%)\end{array}$ & $\begin{array}{l}269 / 85 \\
(31.60 \%)\end{array}$ & $38.0(11.4)$ & $\begin{array}{l}\text { An Internet } \\
\text { intervention on } \\
\text { problem solving } \\
\text { therapy }\end{array}$ & $\begin{array}{l}\text { A self-help book } \\
\text { format without } \\
\text { any form of } \\
\text { guidance. }\end{array}$ & $\mathrm{T}: 8 \mathrm{~W} F: \mathrm{N}$ & $\begin{array}{l}\text { CES-D:I: } \\
27.0(15.1) \\
\text { C:25.9 } \\
(14.9)\end{array}$ \\
\hline $\begin{array}{l}\text { Merry, S. } \\
\text { N.et al./ } \\
\text { New } \\
\text { Zealand/ } \\
2012 \text { [32] }\end{array}$ & $\begin{array}{l}\text { Moderate to } \\
\text { severe } \\
\text { depression } \\
\text { (PHQ-9) }\end{array}$ & $\begin{array}{l}85 / 94 \\
(90.43 \%)\end{array}$ & $\begin{array}{l}187 / 17 \\
(9.09 \%)\end{array}$ & $\begin{array}{l}\text { I:15.55 (1.54) } \\
\text { C:15.58 (1.66) }\end{array}$ & $\begin{array}{l}\text { An interactive fantasy } \\
\text { game (SPARX) }\end{array}$ & $\begin{array}{l}\text { Treatment as } \\
\text { usual }\end{array}$ & $\mathrm{T}: 2 \mathrm{~m} F: 3 \mathrm{~m}$ & $\begin{array}{l}\text { CDRS-R: I: } \\
33.92 \\
(11.19) \mathrm{C:} \\
35.07 \\
(9.71)\end{array}$ \\
\hline $\begin{array}{l}\text { Moritz, S./ } \\
\text { Germany/ } \\
2012[17]\end{array}$ & $\begin{array}{l}\text { depressive } \\
\text { symptoms } \\
\text { (BDI) }\end{array}$ & $\begin{array}{l}82 / 105 \\
(78.10 \%)\end{array}$ & $\begin{array}{l}210 / 40 \\
(19.05 \%)\end{array}$ & $\begin{array}{l}\text { l:38.00 (10.76) } \\
C: 39.13 \\
(15.82)\end{array}$ & $\begin{array}{l}\text { An online self-help pro- } \\
\text { gram for depression } \\
\text { (Deprexis) }\end{array}$ & Waiting list & $\mathrm{T}: 8 \mathrm{~W} F: \mathrm{N}$ & $\begin{array}{l}\text { BDI: :20.51 } \\
(12.22) \mathrm{C:} \\
25.67 \\
(11.65)\end{array}$ \\
\hline
\end{tabular}


Table 1 Details of Included Studies (Continued)

\begin{tabular}{|c|c|c|c|c|c|c|c|c|}
\hline $\begin{array}{l}\text { Author/ } \\
\text { country/ } \\
\text { year }\end{array}$ & Sample type & $\begin{array}{l}\text { Adherence } \\
\text { to the } \\
\text { interventions }\end{array}$ & $\begin{array}{l}\mathrm{N} \\
\text { dropout(\%) }\end{array}$ & $\begin{array}{l}\text { Age } \\
\text { (years,mean) }\end{array}$ & Intervention design & Control group & $\begin{array}{l}\text { Treatment(T) } \\
\& \text { follow } \\
\text { up(F) }\end{array}$ & $\begin{array}{l}\text { Outcome } \\
\text { (mean,SD) }\end{array}$ \\
\hline $\begin{array}{l}\text { Reins, J. } \\
\text { A.et al./ } \\
\text { Germany/ } \\
2019 \text { [18] }\end{array}$ & $\begin{array}{l}\text { major } \\
\text { depressive } \\
\text { disorder } \\
\text { (DSM-IV) }\end{array}$ & $\begin{array}{l}54 / 65 \\
(83.08 \%)\end{array}$ & $\begin{array}{l}131 / 22 \\
(16.80 \%)\end{array}$ & $41.6(10.8)$ & $\begin{array}{l}\text { A guided internet- } \\
\text { based cognitive behav- } \\
\text { ioural therapy (GET.ON } \\
\text { Mood Enhancer) }\end{array}$ & $\begin{array}{l}\text { Online } \\
\text { Psychoeducation } \\
\text { on Depression }\end{array}$ & $\mathrm{T}: 6 \mathrm{w}$ F:3 m & $\begin{array}{l}\text { HRSD: } \\
: 13.75 \\
(7.52) \mathrm{C} \\
16.47 \\
(9.45)\end{array}$ \\
\hline $\begin{array}{l}\text { Roepke, A. } \\
\text { M.et al./the } \\
\text { United } \\
\text { States/2015 } \\
\text { [33] }\end{array}$ & $\begin{array}{l}\text { clinically } \\
\text { significant } \\
\text { depression } \\
\text { (CES-D) }\end{array}$ & $\begin{array}{l}20 / 93 \\
(21.51 \%)\end{array}$ & $\begin{array}{l}186 / 130 \\
(69.89 \%)\end{array}$ & $\begin{array}{l}\text { l:42.28 (12.56) } \\
\text { C:40.27 } \\
(13.06)\end{array}$ & $\begin{array}{l}\text { An innovative } \\
\text { smartphone and } \\
\text { Internet-based game } \\
\text { (SuperBetter) }\end{array}$ & Waiting list & $T: 4 w F: 6 W$ & $\begin{array}{l}\text { CES-D: I: } \\
23.55 \\
(13.73) \mathrm{C}: \\
27.36 \\
(10.63)\end{array}$ \\
\hline $\begin{array}{l}\text { Sander, L. } \\
\text { B.et al./ } \\
\text { Germany/ } \\
2020[34]\end{array}$ & $\begin{array}{l}\text { Mild, } \\
\text { moderate, and } \\
\text { major } \\
\text { depression } \\
\text { (PHQ-9) }\end{array}$ & $\begin{array}{l}102 / 149 \\
(68.46 \%)\end{array}$ & $\begin{array}{l}295 / 71 \\
(24.07 \%)\end{array}$ & $52.8(7.7)$ & $\begin{array}{l}\text { A guided, web-based } \\
\text { self-help intervention } \\
\text { (eSano BackCare-DP) + } \\
\text { automated motivational } \\
\text { text messages }+ \text {-- } \\
\text { coaches give written } \\
\text { feedback to answering } \\
\text { queries. }\end{array}$ & $\begin{array}{l}\text { Treatment as } \\
\text { usual }\end{array}$ & T:9w F:12 m & $\begin{array}{l}\text { HAM-D } \\
\text { score: I: } \\
5.63(3.88) \\
\text { C: } 7.24 \\
(5.38)\end{array}$ \\
\hline $\begin{array}{l}\text { van Luenen, } \\
\text { S. et al./ } \\
\text { Netherlands/ } \\
2018 \text { [35] }\end{array}$ & $\begin{array}{l}\text { mild to } \\
\text { moderate } \\
\text { depressive } \\
\text { symptoms } \\
\text { (PHQ-9) }\end{array}$ & $\begin{array}{l}75 / 97 \\
(77.32 \%)\end{array}$ & $\begin{array}{l}188 / 36 \\
(19.15 \%)\end{array}$ & $46.30(10.63)$ & $\begin{array}{l}\text { An internet-based self- } \\
\text { help intervention con- } \\
\text { sisted of cognitive be- } \\
\text { havioural therapy }\end{array}$ & Waiting list & T:10w F:6 m & $\begin{array}{l}\text { PHQ-9: I: } \\
6.73(3.00) \\
\text { C:8.60 } \\
(3.12)\end{array}$ \\
\hline $\begin{array}{l}\text { Vernmark, K. } \\
\text { et al./ } \\
\text { Sweden/ } \\
2010[36]\end{array}$ & $\begin{array}{l}\text { mild to } \\
\text { moderate } \\
\text { depressive } \\
\text { (MADRS-S) + } \\
\text { major } \\
\text { depression } \\
\text { (DSM-IV) }\end{array}$ & $\begin{array}{l}24 / 27 \\
(88.89 \%)\end{array}$ & $\begin{array}{l}56 / 7 \\
(12.50 \%)\end{array}$ & $36.82(12.9)$ & $\begin{array}{l}\text { An Internet guided self- } \\
\text { help depression pro- } \\
\text { gram: weekly modules } \\
+ \text { homework } \\
\text { assignments }\end{array}$ & Waiting list & $T: 8 w$ F:6 m & $\begin{array}{l}\text { BDI: I: } 12.3 \\
\text { (7.3) C: } \\
16.6(7.9)\end{array}$ \\
\hline $\begin{array}{l}\text { Wilson, } \\
\text { M.et al./the } \\
\text { United } \\
\text { States/2018 } \\
\text { [37] }\end{array}$ & $\begin{array}{l}\text { at least } \\
\text { moderate } \\
\text { depressive } \\
\text { symptoms } \\
\text { (PHQ-9) }\end{array}$ & $\begin{array}{l}22 / 27 \\
(81.48 \%)\end{array}$ & $\begin{array}{l}53 / 6 \\
(11.32 \%)\end{array}$ & $\begin{array}{l}\text { l:45.7 (12.8) C: } \\
47.5(12.9)\end{array}$ & $\begin{array}{l}\text { An internet-based, self- } \\
\text { directed program for } \\
\text { depressive symptoms: } \\
\text { Videos + interactive ac- } \\
\text { tivities + homework } \\
\text { exercises }\end{array}$ & $\begin{array}{l}\text { Treatment as } \\
\text { usual }\end{array}$ & $\mathrm{T}: 4 \mathrm{w} F: 8 \mathrm{w}$ & $\begin{array}{l}\text { PHQ-8: I: } \\
18.40 \\
(5.28) C: \\
20.74 \\
(5.66)\end{array}$ \\
\hline $\begin{array}{l}\text { Zwerenz, } \\
\text { R.et al./ } \\
\text { Germany/ } \\
2019 \text { [38] }\end{array}$ & $\begin{array}{l}\text { at least } \\
\text { moderate } \\
\text { depressive } \\
\text { symptoms } \\
(\mathrm{BDI}-\mathrm{II})+\mathrm{a} \\
\text { clinical } \\
\text { diagnosis of } \\
\text { depression } \\
\text { (ICD-10) }\end{array}$ & $\begin{array}{l}101 / 115 \\
(87.83 \%)\end{array}$ & $\begin{array}{l}229 / 31 \\
(13.54 \%)\end{array}$ & $48(9.79)$ & $\begin{array}{l}\text { A Web-based self-help } \\
\text { program (Deprexis) + } \\
\text { multimodal inpatient } \\
\text { psychodynamic } \\
\text { psychotherapy }\end{array}$ & $\begin{array}{l}\text { An internet } \\
\text { platform provides } \\
\text { basic information } \\
\text { about depression }\end{array}$ & $T: 12 W F: 6 m$ & $\begin{array}{l}\text { BDI-II: I: } \\
18.52 \\
(10.78) \mathrm{C}: \\
24.75 \\
(10.74)\end{array}$ \\
\hline
\end{tabular}

Note. BDI-II Beck Depression Scale II, CES-D The Epidemiology Research Center Depression Scale, PHQ-9 The Patient Health Questionnaire - depression module, PHQ-8 The Patient Health Questionnaire, HRSD/HAMD The Hamilton Depression Scale, HADS Hospital Anxiety and Depression Scale, MADRS-S The MontgomeryEisenberg Depression Scale, CDRS-R A modified childhood depression Rating Scale, CIDI Composite International Diagnostic Interview, DSM-IV Diagnostic and Statistical Manual of Mental Disorders, RADS-2 The Reynolds adolescent depression scale-second edition, QIDS Quick Inventory of Depressive Symptomatology, ICD-10 International Classification of Diseases tenth version

subgroup analysis of the intervention is presented in Additional file 2).

With regard to the different theoretical frameworks on which the intervention was based, the CBT alone group and the CBT combined with other theoretical frameworks group [such as CBT + PST, CBT + positive psychological therapy (PPT), CBT + IPT, and CBT + PPT + $\mathrm{PST}$ ] demonstrated statistically significant improvements in depressive symptoms (CBT alone group: $g=-0.39$;
95\% CI: $-0.58,-0.21 ; p=0.01$; CBT combined with other theoretical frameworks groups: $g=-0.44$; $95 \% \mathrm{CI}$ : $-0.69,-0.19 ; p=0.03)$. The group with PST alone did not display a statistically significant improvement in the depressive symptoms $(g=-0.47 ; 95 \% \mathrm{CI}:-1.17,0.24$; $p=0.00)$. Furthermore, the heterogeneity of the PST alone group was large $\left(\mathrm{I}^{2}=93.25 \%\right)$. However, there was no significant difference among these 3 groups, making it unsuitable for comparison among the groups. 


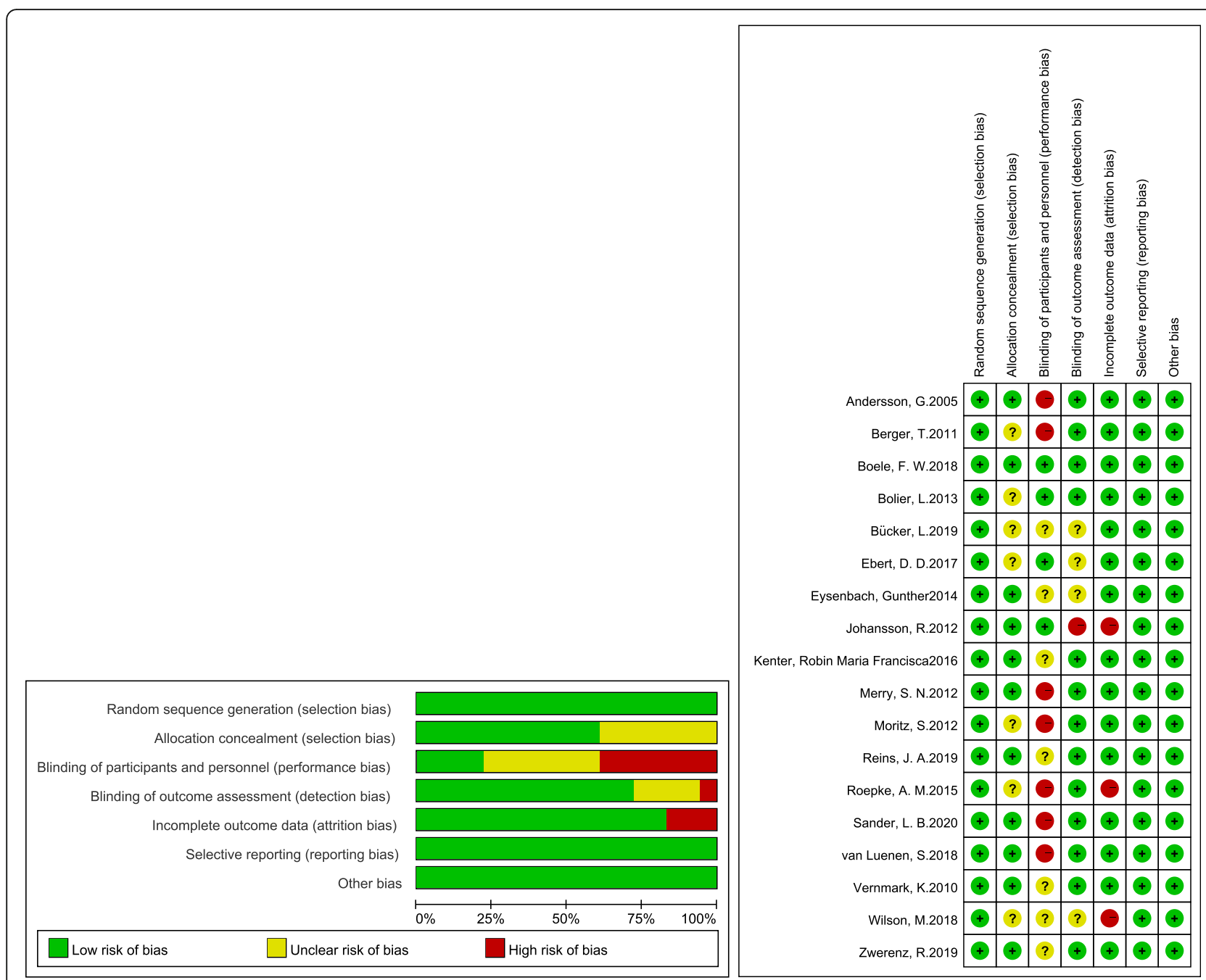

Fig. 2 Quality Assessment of Randomized Controlled Trial: The Risk of Bias Graph and Summary. Note. +, low risk of bias;?, unclear risk of bias; -, high risk of bias

With regard to the intervention duration, the results implied the presence of significant differences in $t \leq 6 \mathrm{w}$ group, $6 \mathrm{w}<\mathrm{t} \leq 8 \mathrm{w}$ group, and $\mathrm{t}>8 \mathrm{w}$ group $(\mathrm{t} \leq 6 \mathrm{w}$ group: $\mathrm{g}=-0.26 ; 95 \% \mathrm{CI}:-0.51,-0.02 ; p=0.22 ; 4 \mathrm{w}<$ $\mathrm{t} \leq 8 \mathrm{w}$ group: $\mathrm{g}=-0.33 ; 95 \% \mathrm{CI}:-0.59,-0.08 ; p=0.00$; $\mathrm{T}>8 \mathrm{w}$ group: $\mathrm{g}=-0.73 ; 95 \% \mathrm{CI}:-0.95,-0.50 ; p=$ $0.03)$. It was further discerned that the longer the intervention time, the higher the improvement in depressive symptoms. The differences among these subgroups were statistically significant $(p=0.01)$.

About the presence of guidance from a therapist, three groups were considered: the group communicating with the therapist, the group not communicating with the therapist, and the group communicating with the virtual healthcare provider. It was found that the group communicating with the therapist exhibited statistically significant improvement in depressive symptoms $(\mathrm{g}=-0.60$; 95\% CI: $-0.81, \quad-0.38 ; \quad p=0.00)$, while the group not communicating with the therapist and the group communicating with the virtual healthcare provider did not exhibit a statistically significant improvement in depressive symptoms $\left(g_{1}=-0.17 ; 95 \%\right.$ CI: $-0.40,0.06 ; \mathrm{p}_{1}=0.20 ; \mathrm{g}_{2}=-$ $0.27 ; 95 \%$ CI: $\left.-0.58,0.05 ; \mathrm{p}_{2}=0.13\right)$. The differences among these subgroups were statistically significant $(p=0.02)$.

Based on the extent of adherence to the intervention, we categorized the subjects into 2 groups: adherence $\leq 90 \%$ and adherence $>90 \%$. The results of subgroup analysis revealed that the intervention effect of high adherence group was better, and the difference between the groups was significant $\left(g_{1}=-0.35 ; 95 \%\right.$ CI: -0.49 , $0.21 ; \mathrm{g}_{2}=-0.97 ; 95 \% \mathrm{CI}:-1.17-0.76 ; p=0.00$ ).

\section{The characteristics of the control group and effects on meta-analysis}

Figure 3C depicts the influence of different control groups on the combined effect size. In our subgroup 


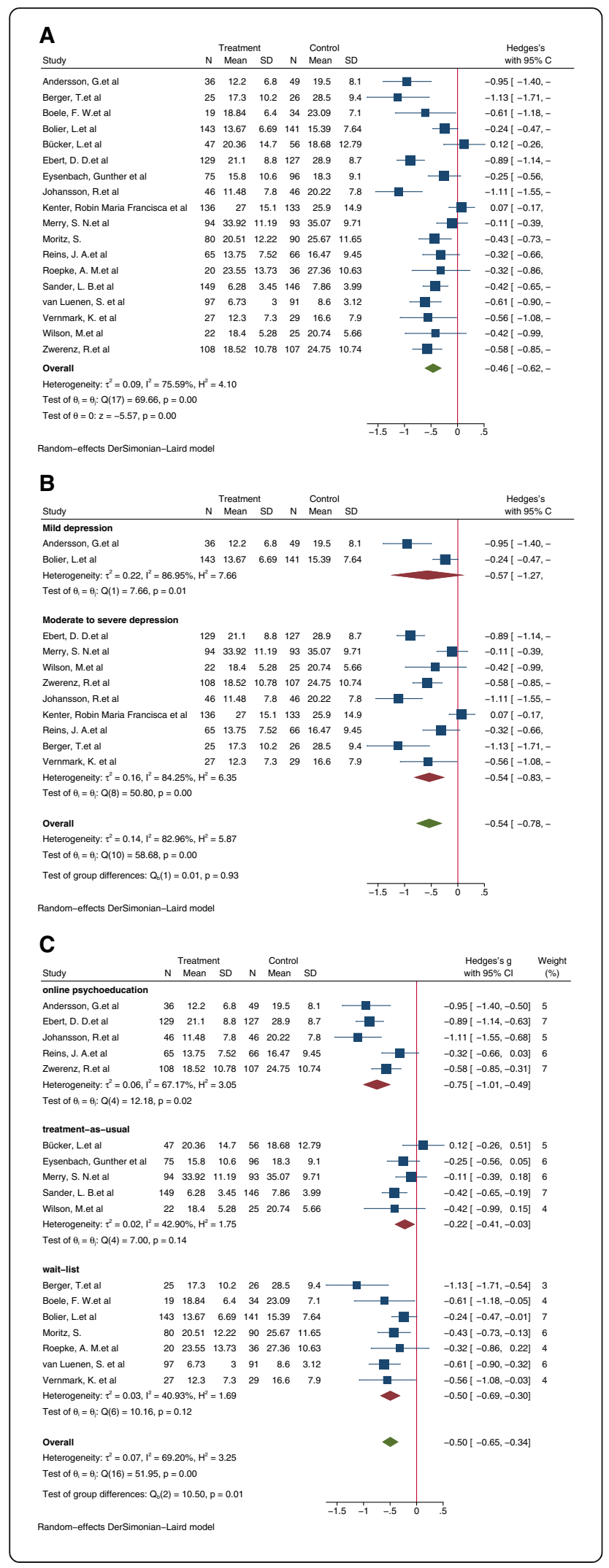

Fig. 3 A Forest Plot of Web-based Self-management versus Control Group for Depression. B Subgroup Analysis: Effectiveness of Webbased Self-management in People with Different Levels of Depression. C Influence of Meta-analysis of Different Control Group

analysis, we identified that the web-based selfmanagement intervention was statistically significant whether compared with the waiting list control group, the treatment-as-usual group or the online psychoeducation group $\left(\mathrm{g}_{1}=-0.50 ; 95 \% \mathrm{CI}:-0.69,-0.30 ; \mathrm{p}_{1}=0.12\right.$; $\mathrm{g}_{2}=-0.22 ; 95 \%$ CI: $-0.41,-0.03 ; \mathrm{p}_{2}=0.14 ; \mathrm{g}_{3}=-0.75$; $\left.95 \% \mathrm{CI}:-1.01,-0.49 ; \mathrm{p}_{3}=0.02\right)$. The online psychoeducation control group showed medium heterogeneity $\left(\mathrm{I}^{2}=67.17 \%\right)$, whereas the heterogeneity of the waiting list and treatment-as-usual control group was small $\left(\mathrm{I}^{2}{ }_{1}=40.93 \% ; \mathrm{I}_{2}^{2}=42.90 \%\right)$. The separate types of control groups were comparable [Q $(2)=10.50, p=0.01]$, and the control group was a significant moderator.

\section{Discussion}

\section{Summary of evidence}

To the best of our knowledge, this is the first metaanalysis exploring the impact of web-based selfmanagement interventions on depressive symptoms. We systematically searched six databases and included 18 randomized controlled trials involving a total of 3055 participants.

Our analyses revealed that web-based self-management interventions exerted a positive effect on depressive symptoms, and there was no indication that publication bias might have influenced the results. We also conducted a subgroup analysis of the control group, and the results revealed that webbased self-management could significantly improve depression when compared with the waiting list, treatment-asusual, or online psychoeducation control groups. The results of the different types of control groups were comparable, and the control group was a significant moderator. This result confirmed the effectiveness of web-based selfmanagement for depression, as well as illustrated that the proposed approach is a potential intervention method.

A meta-analysis [39] has previously been published on the effects of self-management interventions in patients with depression; however, in this article, self-management was intervened by manual approaches. However, our study explored the effectiveness of self-management for depressive symptoms through the use of the Internet. Presently, discussion on which components of web-based self-management interventions play a role in improving depressive symptoms is lacking. However, with the rapid developments in the medical field, literature on psychological intervention via the internet or smartphones has increased dramatically. A metaanalysis [40] has been conducted on the influence of psychological intervention through smartphones on depressive symptoms, and the results asserted that the intervention had 
Table 2 Post-hoc Analyses: Intervention Features

\begin{tabular}{|c|c|c|c|c|c|c|c|c|c|c|}
\hline \multirow[t]{2}{*}{ Aspects of intervention } & \multirow[t]{2}{*}{ Subgroup classification } & \multirow[t]{2}{*}{ Studies } & \multirow[t]{2}{*}{$\begin{array}{l}\text { Sample size } \\
\text { (intervention/ } \\
\text { control) }\end{array}$} & \multicolumn{2}{|c|}{ Meta-analysis } & \multicolumn{3}{|c|}{ Heterogeneity } & \multicolumn{2}{|c|}{$\begin{array}{l}\text { Between } \\
\text { groups } \\
\text { tests }\end{array}$} \\
\hline & & & & $\begin{array}{l}\text { Hedges' } \\
\text { g }\end{array}$ & $95 \% \mathrm{Cl}$ & Q & $p$ & $I^{2}$ & $\mathbf{Q}$ & p \\
\hline \multirow[t]{3}{*}{ Intervention theory } & CBT & 9 & $617 / 645$ & -0.39 & $\begin{array}{l}-0.58,- \\
0.21\end{array}$ & 19.42 & 0.01 & $58.80 \%$ & & \\
\hline & CBT + other therapies & 5 & $371 / 406$ & -0.44 & $\begin{array}{l}-0.69,- \\
0.19\end{array}$ & 10.38 & 0.03 & $61.47 \%$ & & \\
\hline & PST & 3 & $284 / 294$ & -0.47 & $-1.17,0.24$ & 29.64 & 0.00 & $93.25 \%$ & 0.12 & 0.94 \\
\hline \multirow[t]{3}{*}{ Intervention time } & $t \leq 6 w$ & 5 & $173 / 217$ & -0.26 & $\begin{array}{l}-0.51,- \\
0.02\end{array}$ & 5.68 & 0.22 & $29.62 \%$ & & \\
\hline & $6 \mathrm{w}<\mathrm{t} \leq 8 \mathrm{w}$ & 7 & $684 / 709$ & -0.33 & $\begin{array}{l}-0.59 .- \\
0.08\end{array}$ & 33.37 & 0.00 & $82.02 \%$ & & \\
\hline & $t>8 w$ & 6 & $461 / 465$ & -0.73 & $\begin{array}{l}-0.95,- \\
0.50\end{array}$ & 12.69 & 0.03 & $60.61 \%$ & 8.85 & 0.01 \\
\hline \multirow[t]{3}{*}{ Guidance } & Therapist guidance group & 12 & $869 / 909$ & -0.60 & $\begin{array}{l}-0.81,- \\
0.38\end{array}$ & 50.93 & 0.00 & $78.40 \%$ & & \\
\hline & non-therapist guidance group & 3 & $255 / 263$ & -0.17 & $-0.40,0.06$ & 3.21 & 0.20 & $37.65 \%$ & & \\
\hline & $\begin{array}{l}\text { Virtual health indicator guidance } \\
\text { group }\end{array}$ & 2 & $174 / 183$ & -0.27 & $-0.58,0.05$ & 2.29 & 0.13 & $56.30 \%$ & 7.49 & 0.02 \\
\hline \multirow[t]{2}{*}{$\begin{array}{l}\text { Adherence to the } \\
\text { interventions }\end{array}$} & $\leq 90 \%$ & 15 & $1118 / 1192$ & -0.35 & $\begin{array}{l}-0.49,- \\
0.21\end{array}$ & 36.26 & 0.00 & $61.97 \%$ & & \\
\hline & $>90 \%$ & 3 & 200/199 & -0.97 & $\begin{array}{l}-1.17,- \\
0.76\end{array}$ & 1.06 & 0.59 & $0.00 \%$ & 23.60 & 0.00 \\
\hline
\end{tabular}

Note. CBT cognitive behavioral therapy, PST problem-solving treatment

a considerable effect. This finding suggests that informational psychological intervention might be the future trend.

Subgroup analyses of the participant characteristics should be interpreted with caution. Considering the lack of significant difference between the mild and moderateto-severe depression groups, it was impossible to compare between the groups. However, in the moderate-tosevere depression group, the symptoms of the participants were significantly improved, possibly because the patients with moderate-to-severe depression paid more attention to the intervention scheme and better adhered to the prescribed treatment.

A subgroup analysis of intervention theories showed that CBT-based interventions (including CBT alone and CBT combined with other theoretical interventions) significantly reduced depressive symptoms. However, we were unable to clarify which components worked best when the CBT was combined with other theories. A previous study [39] established that CBT-based interventions were more effective in improving the status of depression symptoms than educational interventions. Another review [41] uncovered that interpersonal psychotherapy (IPT) was more effective than other types of psychotherapies. Nonetheless, it is not clear why IPT is more effective than other therapies. A meta-analysis [42] also discerned that different types of psychotherapies (including CBT, IPT, PST, non-directed support therapy, and behavioral activation therapy) were effective in the treatment of adult depression, but no statistically significant difference was observed among the therapies. In our meta-analysis, we noted no significant difference between the groups, making it unsuitable for comparison between the groups. Further research is warranted to compare the advantages and disadvantages of interventions based on the theories established in this study.

In terms of intervention duration, it was found that the standardized mean difference (as Hedges' g) was statistically significant in the $t \leq 6 \mathrm{w}, 6 \mathrm{w}<\mathrm{t} \leq 8 \mathrm{w}$, and $\mathrm{t}>8 \mathrm{w}$ groups. It was also identified that the longer the intervention lasted, the better the improvement in depression. A previous study [43] has explored the difference between short-term and long-term psychotherapies in the treatment of depression. During the 5-year followup, it was noted that the short-term psychotherapy group recovered faster than the long-term psychotherapy group in the beginning. However, when the entire follow-up period was considered, the effect of the intervention on the long-term psychotherapy group was greater than that on the short-term psychotherapy group. This finding is consistent with the results of the present study. Because depression is prone to relapse, self-management needs to be continued for a long time 
to ensure that patients truly master the self-management skills and that they can apply them in their daily lives to achieve the best effect of the intervention.

In terms of intervention function, our subgroup analysis found that the group that communicated with the therapist displayed better improvements in the depressive symptoms than the group that did not communicate with the therapist and the group that communicated with the virtual healthcare provider. The results of a previous systematic review [44], consistent with the outcomes of this study, demonstrated that guided iCBT was associated with more effectiveness than unguided iCBT for individuals with depression. In general, web-based self-management programs without guidance were also effective for depression, although appropriate guidance from psychotherapists was believed to double the positive effects of the intervention. Some past studies have demonstrated that self-management with minimal therapist contact had positive effects on depression [28, 45]. Future research on web-based self-management of depression could be further explored in terms of the contact degree of therapists.

Regarding the status of adherence to the intervention, our results revealed that the intervention effect of the high adherence group was better, and the heterogeneity was small. However, there have been only 3 studies so far with adherence $\geq 90 \%$; therefore, measures to improve treatment adherence should be considered in the research design of related studies in the future.

\section{Limitations}

This meta-analysis has provided important information on the impact of web-based self-management interventions on depressive symptoms. However, certain limitations exist. First, this meta-analysis was heterogeneous. In this study, patients with mild, moderate, and severe depression were included and the interventions were based on various theories, which might have led to the high heterogeneity of the results. However, subgroup analysis was conducted for different conditions to explain the heterogeneity. Second, there were differences in the control group included in this meta-analysis. It included not only the waiting-list group but also the treatment-as-usual group and the online psychoeducation group. Nonetheless, subgroup analysis was carried out in this study. Third, it is difficult to perform further analysis. The studies have obvious design problems such as methodological flaws, high-risk allocation order, diverse measurement methods, lack of follow-up data, and difficulty in intervention control. Finally, psychotherapy is a long-term rehabilitation process, and the short-term effects may not be significant. The intervention time in this paper was short, and there was no long-term follow- up. Hence, further research is needed to clarify the longterm effects and the efficacy of the intervention.

\section{Conclusions}

In summary, comprehensive evidence suggests that webbased self-management interventions can alleviate depressive symptoms. However, treatment via the internet is associated with challenges such as failure to ensure the patients' adherence to the treatment, high rate of loss of follow-up, failure to determine the long-term effects of intervention, and the impact of individual depression severity on the effectiveness of the intervention. Future research is required to optimize and personalize web-based self-management interventions for depression.

As technology continues to advance and web-based self-management interventions for depression prove to be effective, the number of empirical studies in this field is growing rapidly. It could be stated that this kind of informational depression intervention is likely to be the future trend. Therefore, while continuing to design and evaluate the best web-based interventions, further research should be undertaken to develop viable approaches for implementing web-based interventions in healthcare systems.

\section{Supplementary Information}

The online version contains supplementary material available at https://doi. org/10.1186/s12888-021-03396-8.

Additional file 1. Search strategy

Additional file 2. Subgroup analysis: Forest plot of intervention theory, time, adherence, and whether or not to communicate with the therapist.

Additional file 3. Publication bias (Egger's test and Trim-and-fill analysis).

\begin{abstract}
Acknowledgments
The author would like to acknowledge the Postgraduate Innovation Research Program of Jilin University for its funding. The authors would like to thank all the reviewers who participated in the review and to MJEditor (www.mjeditor.com) for its linguistic assistance during the preparation of this manuscript.
\end{abstract}

\section{Authors' contributions}

Study design: YP, FL. Literature search: XZ. Data collection: LX. Analysis and interpretation of data: RG, MS. Manuscript preparation: HS. Drafting the manuscript: YP. Review of the manuscript: YL. The author(s) read and approved the final manuscript.

\section{Funding}

2020 Postgraduate Innovation Research Program of Jilin University (Project ref.: 101832020(X323).

\section{Availability of data and materials}

The data that support the findings of this study are available on request from the corresponding author. The data are not publicly available due to privacy or ethical restrictions. 


\section{Declarations}

Ethics approval and consent to participate

Not applicable.

\section{Consent for publication}

Not applicable.

\section{Competing interests}

The author has no conflicts of interest.

\section{Received: 8 March 2021 Accepted: 26 July 2021}

Published online: 11 August 2021

\section{References}

1. Kessler RC, Bromet EJ. The epidemiology of depression across cultures. Annu Rev Public Health. 2013;34(1):119-38. https://doi.org/10.1146/annurevpublhealth-031912-114409.

2. Bromet E, Andrade LH, Hwang I, Sampson NA, Alonso J, de Girolamo G, et al. Cross-national epidemiology of DSM-IV major depressive episode. BMC Med. 2011;9(1):90. https://doi.org/10.1186/1741-7015-9-90.

3. Malhi GS, Outhred T, Hamilton A, Boyce PM, Bryant R, Fitzgerald PB, et al. Royal Australian and new Zealand College of Psychiatrists clinical practice guidelines for mood disorders: major depression summary. Med J Aust. 2018;208(4):175-80. https://doi.org/10.5694/mja17.00659.

4. McCrone PR, Dhanasiri S, Patel A, et al. Paying the price: the cost of mental health care in England to 2026. London: King's Fund; 2008.

5. Ali S, Stone MA, Peters JL, Davies MJ, Khunti K. The prevalence of co-morbid depression in adults with type 2 diabetes: a systematic review and metaanalysis. Diabet Med. 2006;23(11):1165-73. 17054590. https://doi.org/1 0.1111/j.1464-5491.2006.01943.x.

6. Sun WJ, Xu L, Chan WM, Lam TH, Schooling CM. Schooling CM. Depressive symptoms and suicide in 56,000 older Chinese: a Hong Kong cohort study. Soc Psychiatry Psychiatr Epidemiol. 2012;47(4):505-14. https://doi.org/10.1 007/s00127-011-0362-z.

7. Curtin SC, Warner M, Hedegaard H. Increase in Suicide in the United States, 1999-2014. NCHS Data Brief. 2016:(241):1-8.

8. Gonzalez HM, Vega WA, Williams DR, et al. Depression care in the United States: too little for too few. Arch Gen Psychiatry. 2010;67:37-46.

9. Cuijpers P, Sijbrandij M, Koole SL, Andersson G, Beekman AT, Reynolds CF III. The efficacy of psychotherapy and pharmacotherapy in treating depressive and anxiety disorders: a meta-analysis of direct comparisons. World Psychiatry. 2013;12(2):137-48. https://doi.org/10.1002/wps.20038.

10. World Health Organization. Global health workforce, finances remain low for mental health. 2015. www.who.int. Accessed 2 Dec 2020.

11. Liu JX, Goryakin Y, Maeda A, Bruckner T, Scheffler R. Global health workforce labor market projections for 2030. Hum Resour Health. 2017;15(1):11. https:// doi.org/10.1186/s12960-017-0187-2

12. Fricchione GL, Borba CP, Alem A, et al. Capacity building in global mental health: professional training. Harv Rev Psychiatry. 2012;20(1):47-57. https:// doi.org/10.3109/10673229.2012.655211.

13. Duggal HS. Self-Management of Depression: Beyond the Medical Model. Perm J. 2019;23:18-295. https://doi.org/10.7812/TPP/18-295. Epub 2019 May 31. PMID: $31167709 ;$ PMCID: PMC6550311.

14. Andersson G. Using the internet to provide cognitive behaviour therapy. Behav Res Ther. 2009;47(3):175-80. https://doi.org/10.1016/j.brat.2009.01.010.

15. Johansson R, Ekbladh S, Hebert A, et al. Psychodynamic guided self-help for adult depression through the internet: a randomised controlled trial. PLoS One. 2012;7(5):e38021. https://doi.org/10.1371/journal.pone.0038021 Epub 2012 May 29. PMID: 22741027; PMCID: PMC3362510.

16. Ebert DD, Nobis S, Lehr D, Baumeister H, Riper H, Auerbach RP, et al. The 6month effectiveness of internet-based guided self-help for depression in adults with type 1 and 2 diabetes mellitus. Diabet Med. 2017;34(1):99-107. https://doi.org/10.1111/dme.13173 Epub 2016 Aug 4. PMID: 27334444

17. Moritz S, Schilling L, Hauschildt M, Schröder J, Treszl A. A randomized controlled trial of internet-based therapy in depression. Behav Res Ther. 2012;50(7-8):513-21. https://doi.org/10.1016/j.brat.2012.04.006 Epub 2012 May 3. PMID: 22677231.

18. Reins JA, Boß L, Lehr D, Berking M, Ebert DD. The more I got, the less I need? Efficacy of internet-based guided self-help compared to online psychoeducation for major depressive disorder. J Affect Disord. 2019;246:
695-705. https://doi.org/10.1016/j.jad.2018.12.065 Epub 2018 Dec 21. PMID: 30611913.

19. Andersson G, Hesser H, Veilord A, Svedling L, Andersson F, Sleman O, et al. Randomised controlled non-inferiority trial with 3-year follow-up of internetdelivered versus face-to-face group cognitive behavioural therapy for depression. J Affect Disord. 2013;151(3):986-94. https://doi.org/10.1016/j.ja d.2013.08.022 Epub 2013 Aug 30. PMID: 24035673.

20. Hunkeler EM, Hargreaves WA, Fireman B, Terdiman J, Meresman JF, Porterfield $Y$, et al. A web-delivered care management and patient selfmanagement program for recurrent depression: a randomized trial. Psychiatr Serv. 2012;63(11):1063-71. https://doi.org/10.1176/appi.ps.005332 011 PMID: 22983558.

21. Boele FW, Klein M, Verdonck-de Leeuw IM, et al. Internet-based guided selfhelp for glioma patients with depressive symptoms: a randomized controlled trial. J Neurooncol. 2018;137(1):191-203. https://doi.org/10.1007/ s1 1060-017-2712-5 Epub 2017 Dec 13. PMID: 29236238; PMCID: PMC5846975.

22. Geraedts AS, Kleiboer AM, Twisk J, et al. Long-term results of a web-based guided self-help intervention for employees with depressive symptoms: randomized controlled trial. J Med Internet Res. 2014;16(7):e168. https://doi. org/10.2196/jmir.3539 PMID: 25008127; PMCID: PMC4115257.

23. Kenter RM, Cuijpers $P$, Beekman A, et al. Effectiveness of a Web-Based Guided Self-help Intervention for Outpatients With a Depressive Disorder: Short-term Results From a Randomized Controlled Trial. J Med Internet Res. 2016;18(3):e80. https://doi.org/10.2196/jmir.4861 PMID: 27032449; PMCID: PMC4832120.

24. Karyotaki E, Riper H, Twisk J, Hoogendoorn A, Kleiboer A, Mira A, et al. Efficacy of self-guided internet-based cognitive behavioral therapy in the treatment of depressive symptoms: a meta-analysis of individual participant data. JAMA Psychiatry. 2017;74(4):351-9. 28241179. https://doi.org/10.1001/ja mapsychiatry.2017.0044.

25. Liberati A, Altman DG, Tetzlaff J, Mulrow C, Gotzsche PC, loannidis JPA, et al. The PRISMA statement for reporting systematic reviews and meta-analyses of studies that evaluate healthcare interventions: explanation and elaboration. BMJ. 2009;339(jul21 1):b2700. https://doi.org/10.1136/bmj.b2700.

26. Moher D, Liberati A, Tetzlaff J, Altman DG, The PRISMA Group. Preferred reporting items for systematic reviews and meta-analyses: the PRISMA statement. PLoS Med. 2009;6(7):e1000097. https://doi.org/10.1371/journal. pmed.1000097.

27. Higgins JP, Altman DG, Gøtzsche PC, et al. The Cochrane Collaboration's tool for assessing risk of bias in randomised trials. BMJ. 2011;343(oct18 2): d5928. https://doi.org/10.1136/bmj.d5928.

28. Andersson G, Bergström J, Holländare F, Carlbring P, Kaldo V, Ekselius L. Internetbased self-help for depression: randomised controlled trial. Br J Psychiatry. 2005; 187(5):456-61. 16260822. https//doi.org/10.1192/bjp.187.5.456.

29. Berger T, Hämmerli K, Gubser N, Andersson G, Caspar F. Internet-based treatment of depression: a randomized controlled trial comparing guided with unguided self-help. Cogn Behav Ther. 2011;40(4):251-66. 22060248. https://doi.org/10.1080/16506073.2011.616531.

30. Bolier L, Haverman M, Kramer J, et al. An Internet-based intervention to promote mental fitness for mildly depressed adults: randomized controlled trial. J Med Internet Res. 2013;15(9):e200. https://doi.org/10.2196/jmir.2603 PMID: 24041479; PMCID: PMC3929047.

31. Bücker L, Schnakenberg P, Karyotaki E, et al. Diminishing Effects After Recurrent Use of Self-Guided Internet-Based Interventions in Depression: Randomized Controlled Trial. J Med Internet Res. 2019;21(10):e14240. https://doi.org/10.2196/14240 PMID: 31579014; PMCID: PMC6777284.

32. Merry SN, Stasiak K, Shepherd M, et al. The effectiveness of SPARX, a computerised self help intervention for adolescents seeking help for depression: randomised controlled non-inferiority trial. BMJ. 2012:344e2598. https://doi.org/10.1136/bmj.e2598 PMID: 22517917; PMCID: PMC3330131.

33. Roepke AM, Jaffee SR, Riffle OM, McGonigal J, Broome R, Maxwell B. Randomized controlled trial of SuperBetter, a smartphone-based/internetbased self-help tool to reduce depressive symptoms. Games Health J. 2015; 4(3):235-46. https://doi.org/10.1089/g4h.2014.0046 Epub 2015 Feb 19. PMID: 26182069.

34. Sander $L B$, Paganini $S$, Terhorst $Y$, et al. Effectiveness of a Guided Web-Based Self-help Intervention to Prevent Depression in Patients With Persistent Back Pain: The PROD-BP Randomized Clinical Trial. JAMA Psychiatry. 2020;77(10): 
1001-11. https://doi.org/10.1001/jamapsychiatry.2020.1021 PMID: 32459348; PMCID: PMC7254449.

35. van Luenen S, Garnefski N, Spinhoven P, et al. Guided internet-based intervention for people with HIV and depressive symptoms: a randomised controlled trial in the Netherlands. Lancet HIV. 2018;5(9):e488-97. https://doi. org/10.1016/S2352-3018(18)30133-4 Epub 2018 Aug 19. Erratum in: Lancet HIV. 2018 Oct;5(10):e545. PMID: 30135045.

36. Vernmark K, Lenndin J, Bjärehed J, Carlsson M, Karlsson J, Öberg J, et al. Internet administered guided self-help versus individualized e-mail therapy: a randomized trial of two versions of CBT for major depression. Behav Res Ther. 2010;48(5):368-76. https://doi.org/10.1016/j.brat.2010.01.005 Epub 2010 Feb 2. PMID: 20152960.

37. Wilson M, Hewes C, Barbosa-Leiker C, Mason A, Wuestney KA, Shuen JA, et al. Engaging adults with chronic disease in online depressive symptom self-management. West J Nurs Res. 2018;40(6):834-53. https://doi.org/10.11 77/0193945916689068 Epub 2017 Jan 30. PMID: 28322662.

38. Zwerenz R, Baumgarten C, Becker J, et al. Improving the Course of Depressive Symptoms After Inpatient Psychotherapy Using Adjunct WebBased Self-Help: Follow-Up Results of a Randomized Controlled Trial. J Med Internet Res. 2019;21(10):e13655. https://doi.org/10.2196/13655 PMID: 31651403; PMCID: PMC6838691.

39. Gellatly J, Bower P, Hennessy S, et al. What makes self-help interventions effective in the management of depressive symptoms? Meta-analysis and meta-regression. Psychol Med. 2007;37(9):1217-28. https://doi.org/10.1017/ S0033291707000062 Epub 2007 Feb 19. PMID: 17306044.

40. Firth J, Torous J, Nicholas J, Carney R, Pratap A, Rosenbaum S, et al. The efficacy of smartphone-based mental health interventions for depressive symptoms: a meta-analysis of randomized controlled trials. World Psychiatry. 2017;16(3):287-98. https://doi.org/10.1002/wps.20472.

41. Cuijpers P, van Straten A, Andersson G, van Oppen P. Psychotherapy for depression in adults: a meta-analysis of comparative outcome studies. J Consult Clin Psychol. 2008;76(6):909-22. 19045960. https://doi.org/10.1037/a 0013075.

42. Cuijpers P, Andersson G, Donker T, van Straten A. Psychological treatment of depression: results of a series of meta-analyses. Nord J Psychiatry. 2011; 65(6):354-64. https://doi.org/10.3109/08039488.2011.596570 Epub $2011 \mathrm{Jul}$ 20. PMID: 21770842

43. Maljanen $T$, Knekt $P$, Lindfors $\mathrm{O}$, Virtala E, Tillman P, Härkänen $T$, et al. The cost-effectiveness of short-term and long-term psychotherapy in the treatment of depressive and anxiety disorders during a 5-year follow-up. J Affect Disord. 2016;190:254-63. https://doi.org/10.1016/j.jad.2015.09.065 Epub 2015 Oct 24. PMID: 26540079

44. Karyotaki E, Efthimiou O, Miguel C, et al. Internet-Based Cognitive Behavioral Therapy for Depression: A Systematic Review and Individual Patient Data Network Meta-analysis. JAMA Psychiatry. 2021;78(4):361-371. doi: https://doi. org/10.1001/jamapsychiatry.2020.4364. PMID: 33471111; PMCID: PMC8027916.

45. Arean PA, Hallgren KA, Jordan JT, et al. The Use and Effectiveness of Mobile Apps for Depression: Results From a Fully Remote Clinical Trial. J Med Internet Res. 2016;18(12):e330. doi: https://doi.org/10.2196/jmir.6482. PMID: 27998876; PMCID: PMC5209607.

\section{Publisher's Note}

Springer Nature remains neutral with regard to jurisdictional claims in published maps and institutional affiliations.

Ready to submit your research? Choose BMC and benefit from:

- fast, convenient online submission

- thorough peer review by experienced researchers in your field

- rapid publication on acceptance

- support for research data, including large and complex data types

- gold Open Access which fosters wider collaboration and increased citations

- maximum visibility for your research: over $100 \mathrm{M}$ website views per year

At BMC, research is always in progress.

Learn more biomedcentral.com/submissions 